Departamento de Ciência e Tecnologia, Secretaria de Ciência, Tecnologia e Insumos Estratégicos, Ministério da Saúde

Correspondência | Correspondence: Decit - Departamento de Ciência e Tecnologia do Ministério da Saúde

Esplanada dos Ministérios

Bloco G sala 845

70058-900 Brasília, DF, Brasil

Texto de difusão técnico-científica do Ministério de Saúde.

\section{Avaliação nacional do Programa de Humanização do Pré-natal e Nascimento}

\section{National evaluation of the Humanization of Prenatal and Birth Program}

A avaliação nacional do Programa de Humanização do Pré-natal e Nascimento (PHPN) foi o primeiro projeto de pesquisa financiado diretamente pelo Departamento de Ciência e Tecnologia (Decit)/Ministério da Saúde (MS), por meio do Edital 252/2002, implementado pela Organização das Nações Unidas para a Educação, a Ciência e a Cultura (Unesco). O projeto, aprovado em concorrência pública, foi realizado pela equipe de pesquisadores da Faculdade de Saúde Pública da Universidade de São Paulo. ${ }^{a}$ desenvolvido no período de 12 meses, iniciando em janeiro de 2003.

O objetivo era avaliar o processo de implantação e implementação do PHPN, desenvolvido pela Área Técnica de Saúde da Mulher do Ministério da Saúde.

A metodologia da pesquisa utilizou as abordagens quantitativas e qualitativas de avaliação, tendo como unidade de análise cada município do Brasil.

A avaliação iniciou-se pela análise dos dados quantitativos do sistema Datasus, base de dados demográfi$\cos ^{\mathrm{b}}$ e os relatórios gerenciais do software Sistema de Acompanhamento do Programa de Humanização no Pré-Natal e Nascimento (Sisprenatal). Os municípios foram agrupados em clusters por porte populacional e por região geográfica do País. Buscou-se identificar os fatores intervenientes que influenciaram a execução do Programa, tanto do contexto local como nacional, bem como os fatores preditivos de maior probabilidade de desempenho positivo do PHPN. Para a análise de correlações foi utilizado o teste paramétrico de Pearson.

Com base na análise quantitativa, foi possível formular hipóteses de relação entre as variáveis de contexto porte populacional, existência de recursos ambulatoriais e/ou hospitalares, Programa de Agente Comunitário de Saúde (PACS), Programa de Saúde da Família (PSF), e outras - com indicadores de desempenho, selecionados a partir do Sisprenatal.
Os municípios de distintos portes e regiões do País foram selecionados de acordo com o estágio de desenvolvimento do PHPN. Os critérios utilizados para seleção foram: 1. relações significativas entre as variáveis de contexto favorecedoras do desempenho apresentado no PHPN; 2. municípios que, apesar de apresentarem todas as condições favoráveis, apresentavam desempenho contraditoriamente insuficiente. Deste conjunto foram selecionados os casos para o estudo de campo, de forma conjunta e consensual com técnicos da Coordenação Nacional de Saúde da Mulher/MS e do Decit/MS.

A abordagem qualitativa de estudo de caso foi aplicada a cada um dos municípios selecionados. Os dados primários foram coletados por meio de entrevistas semiestruturadas com informantes-chave (profissionais) e grupos focais com usuárias dos serviços de saúde. Esses dados foram trabalhados utilizando a análise temática e exploradas as seguintes características: organização do município, central de regulação e mecanismo de vinculação da gestante.

Os dados quantitativos mostraram que dos $5.561 \mathrm{mu}-$ nicípios brasileiros, em 2002, 3.923 haviam aderido ao PHPN. Destes, 2.031 apresentavam algum registro de produção do Programa e somente 634 apresentavam registro no Sisprenatal com algumas gestantes com todos os procedimentos previstos no PHPN. O total de gestantes cadastradas no País entre janeiro de 2001 a agosto de 2002 era de 316.154 mulheres.

A Tabela 1 mostra a análise dos municípios quanto à realização de todas as atividades programadas para cobrir o ciclo gravídico puerperal completo, por porte de município e condição de adesão ao Programa. Os dados mostram que a realização dos procedimentos previstos no PHPN são mais freqüentes nos municípios de grande porte, indicando que a oferta de serviços existentes é um elemento facilitador no cumprimento do programado.

\footnotetext{
a A equipe é constituída por Oswaldo Yoshimi Tanaka (coordenador), Ana Cristina d’Andretta Tanaka, Fernão Dias de Lima, Daniel Marucci,

Carmen A. C. Carvalho, Cristiane Locatelli, Edith L. Ribeiro, Marilia L. A. Ohta.

${ }^{\text {b }}$ Fundação Sistema Estadual de Análise de Dados. Disponível em www.seade.gov.br
} 
Ao se analisar o tempo de adesão ao PHPN (Tabela 2), verifica-se que é inversamente proporcional ao porte do município, à semelhança do tempo entre a

Tabela 1. Distribuição dos municípios brasileiros, segundo porte, adesão ao programa de humanização do pré-natal e produção de procedimentos completos. Brasil, 2002.

\begin{tabular}{|c|c|c|c|}
\hline \multirow[t]{2}{*}{ Porte (habitantes) } & \multicolumn{3}{|c|}{$\begin{array}{l}\text { Realização de procedimentos } \\
\text { completos }\end{array}$} \\
\hline & Não adesão & Adesão & Total \\
\hline$<20.000$ & 1.005 & 616 & 1.621 \\
\hline 20.000 a 49.999 & 255 & 206 & 461 \\
\hline$\geq 50.000$ & 128 & 185 & 313 \\
\hline Capitais & 8 & 18 & 26 \\
\hline Total & 1.396 & 1.025 & 2.421 \\
\hline
\end{tabular}

Percentuais sobre o porte

$\begin{array}{llll}<20.000 & 62,0 & 38,0 & 100,0 \\ 20.000 \text { a 49.999 } & 55,3 & 44,7 & 100,0 \\ \geq 50.000 & 40,9 & 59,1 & 100,0 \\ \text { Capitais } & 30,8 & 69,2 & 100,0 \\ \text { Total } & 57,7 & 42,3 & 100,0\end{array}$

Percentuais sobre a produção

\begin{tabular}{cccc}
$<20.000$ & 72,0 & 60,1 & 67,0 \\
20.000 a 49.999 & 18,3 & 20,1 & 19,0 \\
$\geq 50.000$ & 9,2 & 18,0 & 12,9 \\
Capitais & 0,6 & 1,8 & 1,1 \\
\hline Total & 100,0 & 100,0 & 100,0 \\
\hline
\end{tabular}

adesão e o início dos procedimentos. Dentre os municípios de grande porte, as capitais representaram uma exceção, apresentando o maior tempo necessário para a adesão. Embora os municípios de pequeno porte demorem mais para aderir e iniciar os procedimentos, eles completam o Programa em menor tempo que os municípios de grande porte.

Esses resultados permitem levantar a hipótese de que os municípios menores encontram dificuldades na operacionalização do PHPN, relacionados a recursos humanos e capacidade técnica necessária para desencadear a organização dos dados, pactuações e recursos para o planejamento do sistema de saúde local.

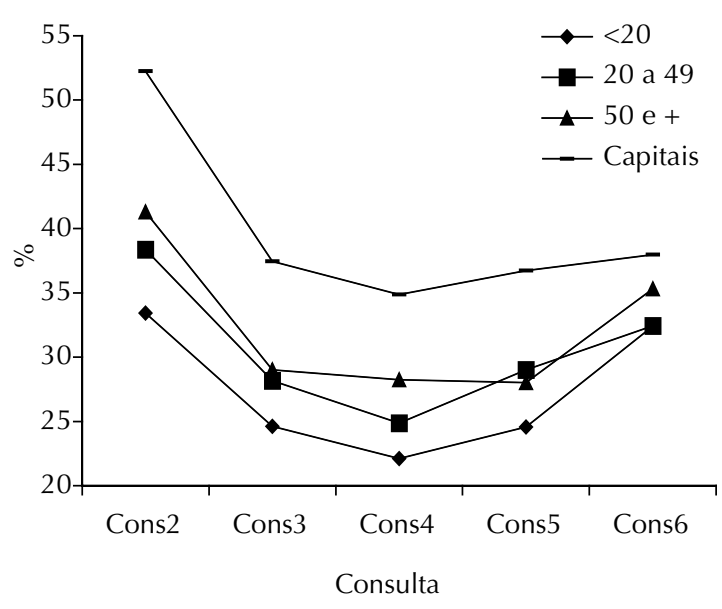

Figura 1. Média de evasão de gestantes em relação à consulta anterior, segundo porte dos municípios. Brasil, 2002.

Tabela 2. Distribuição dos municípios brasileiros, segundo o porte e o tempo de adesão ao programa de humanização do pré-natal. Brasil, 2002.

\begin{tabular}{lcccccc}
\hline Porte (habitantes) & $\mathrm{N}$ & Média meses & Desvio-padrão & Erro-padrão & Mínimo & Máximo \\
\hline$<20.000$ & 2.818 & 13,64 & 5,18 & 0,10 & 2 & 25 \\
20.000 a 49.999 & 681 & 13,54 & 5,16 & 0,20 & 2 & 25 \\
$\geq 50.000$ & 397 & 12,50 & 5,03 & 0,25 & 0 & 25 \\
Capitais & 27 & 9,78 & 4,97 & 0,96 & 0 & 22 \\
\hline Total & 3.923 & 13,48 & 5,18 & 0,08 & 0 & 25 \\
\hline
\end{tabular}

Tabela 3. Distribuição dos municípios brasileiros com base no indicador de produtividade, segundo o porte e a produção de procedimentos completos. Brasil, 2000-2001.

\begin{tabular}{lccccc}
\hline Porte (habitantes) & $\mathrm{N}$ & Média & Mediana & DP & Relação mediana \\
\hline$<20.000$ & 617 & 11,84 & 5,27 & 18,72 & 1,00 \\
20.000 a 49.999 & 206 & 37,22 & 17,61 & 63,15 & 3,34 \\
$\geq 50.000$ & 185 & 110,25 & 45,81 & 176,15 & 8,69 \\
Capitais & 18 & 1060,10 & 444,76 & 1931,66 & 84,39 \\
\hline Total & 1026 & 53,07 & 9,33 & 296,54 & \\
\hline
\end{tabular}




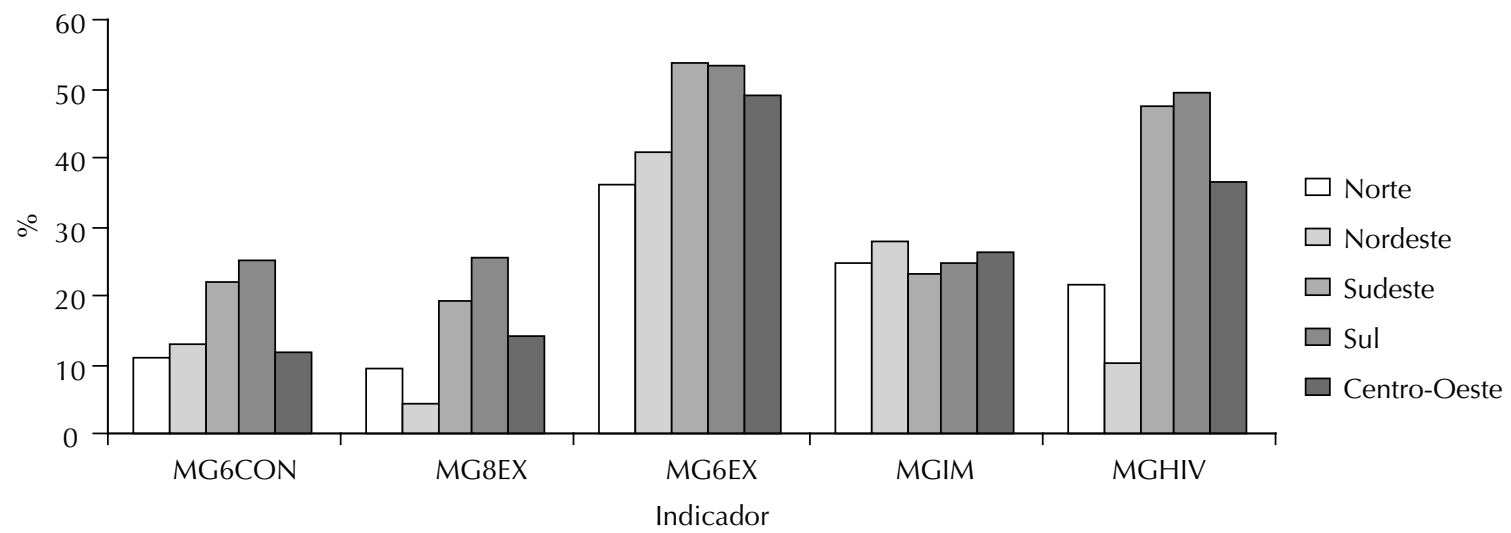

MG6CON: gestantes com seis consultas de pré-natal

MG8EX: gestantes com oito exames laboratoriais

MG6EX: gestantes com seis exames laboratoriais

MGIM: gestantes imunizadas

MGHIV: gestantes com exame HIV

Figura 2. Média percentual dos principais indicadores de atenção, segundo região. Brasil, 2002.

Tabela 4. Percentual de adesão ao Programa de Saúde da Família segundo condição e tempo de adesão ao programa de humanização do pré-natal, por porte dos municípios. Brasil, 2002.

\begin{tabular}{|c|c|c|c|c|c|}
\hline Porte (habitantes) & $\mathrm{N}$ & Média/meses & Mediana & DP & $\mathrm{p}$ \\
\hline \multicolumn{6}{|l|}{ Não adesão } \\
\hline$<20.000$ & 1.254 & 6,01 & 0,00 & 20,22 & 0,0000 \\
\hline 20.000 a 49.999 & 283 & 4,38 & 0,00 & 13,92 & 0,0000 \\
\hline$\geq 50.000$ & 101 & 3,50 & 0,00 & 8,06 & 0,0247 \\
\hline \multicolumn{6}{|l|}{ Adesão } \\
\hline$<20.000$ & 2.818 & 13,20 & 0,00 & 29,26 & \\
\hline 20.000 a 49.999 & 681 & 9,08 & 0,00 & 19,29 & \\
\hline$\geq 50.000$ & 397 & 6,89 & 0,00 & 14,56 & \\
\hline Capitais & 27 & 4,80 & 0,77 & 10,14 & \\
\hline Total & 5.561 & 9,96 & 0,00 & 24,70 & \\
\hline
\end{tabular}

Tabela 5. Indicador de recursos ambulatoriais segundo condição de adesão ao PHPN e a região. Brasil, 2002.

\begin{tabular}{|c|c|c|c|c|c|c|}
\hline Adesão/Região & $\mathrm{N}$ & Média & Mediana & DP & Mínimo & Máximo \\
\hline \multicolumn{7}{|l|}{ Não } \\
\hline Norte & 155 & 5,26 & 3,24 & 6,28 & 0,10 & 40,60 \\
\hline Nordeste & 640 & 4,73 & 3,28 & 4,99 & 0,04 & 63,32 \\
\hline Sudeste & 401 & 3,68 & 2,66 & 4,26 & 0,12 & 34,50 \\
\hline Sul & 375 & 2,72 & 1,35 & 5,86 & 0,04 & 84,42 \\
\hline Centro-Oeste & 32 & 4,31 & 2,45 & 4,75 & 0,08 & 20,67 \\
\hline \multicolumn{7}{|l|}{ Sim } \\
\hline Norte & 286 & 4,94 & 3,03 & 5,61 & 0,04 & 34,45 \\
\hline Nordeste & 1149 & 3,96 & 3,22 & 3,14 & 0,13 & 30,02 \\
\hline Sudeste & 1232 & 3,67 & 2,50 & 3,88 & 0,03 & 41,27 \\
\hline Sul & 792 & 2,40 & 1,72 & 2,60 & 0,03 & 28,63 \\
\hline Centro-Oeste & 417 & 3,33 & 2,20 & 3,82 & 0,06 & 35,71 \\
\hline Total & 5479 & 3,70 & 2,53 & 4,19 & 0,03 & 84,42 \\
\hline
\end{tabular}


Tabela 6. Correlações encontradas e que vão ao encontro das hipóteses, por porte do município e região, de acordo com as variáveis facilitadoras e o tipo de procedimento. Brasil, 2002.

\begin{tabular}{|c|c|c|c|c|c|c|c|c|c|c|c|c|c|}
\hline \multirow[b]{2}{*}{ Porte (habitantes) } & \multirow[b]{2}{*}{ Var.Facilit. } & \multicolumn{2}{|c|}{ Norte } & \multicolumn{2}{|c|}{ Nordeste } & \multicolumn{2}{|c|}{$\begin{array}{l}\text { Região / } \\
\text { Sudeste }\end{array}$} & \multicolumn{2}{|c|}{$\begin{array}{l}\text { Procedi- } \\
\text { mento Sul }\end{array}$} & \multicolumn{2}{|c|}{$\begin{array}{c}\text { Centro- } \\
\text { Oeste }\end{array}$} & \multicolumn{2}{|c|}{ Total } \\
\hline & & P102 & P103 & P102 & P103 & P102 & P103 & P102 & P103 & P102 & P103 & P102 & P103 \\
\hline \multirow{5}{*}{$<20.000$} & $\mathrm{PN}$ & & & & & & & & & & & & \\
\hline & RA & & & & & & & & & & & & \\
\hline & $\mathrm{RH}$ & & & & & & 1 & & & & 2 & & 1 \\
\hline & $\mathrm{AC}$ & & 3 & & 1 & 1 & & & & & 5 & & \\
\hline & $\mathrm{AF}$ & 2 & 4 & 2 & & & 4 & & 2 & & & 1 & 1 \\
\hline \multirow[t]{5}{*}{20.000 a 49.999} & $\mathrm{PN}$ & & 8 & & & & & & & & & & \\
\hline & RA & & & 3 & 2 & & 2 & 6 & & 1 & & 3 & 1 \\
\hline & $\mathrm{RH}$ & 3 & & & & & 2 & & 1 & & & & 3 \\
\hline & $\mathrm{AC}$ & 3 & 6 & & & & & & & & 1 & & \\
\hline & $\mathrm{AF}$ & & & & & & & 2 & & 1 & & & \\
\hline \multirow[t]{5}{*}{$\geq 50.000$} & $\mathrm{PN}$ & & & & & & & & & & & & \\
\hline & RA & 8 & 6 & 2 & 2 & 1 & & 2 & & & & & 1 \\
\hline & $\mathrm{RH}$ & & 7 & & 1 & & & & & & & & \\
\hline & $\mathrm{AC}$ & & & & 2 & 2 & & 1 & 1 & 5 & 5 & & 1 \\
\hline & $\mathrm{AF}$ & & & 1 & 3 & 1 & & 3 & & & & & 1 \\
\hline \multirow[t]{5}{*}{ Capitais } & PN & & & & & 3 & & & & & & & 2 \\
\hline & RA & 5 & & 2 & & 8 & & & & 3 & 3 & 5 & 3 \\
\hline & $\mathrm{RH}$ & & & 5 & & 4 & & 6 & & 5 & 4 & 1 & \\
\hline & $A C$ & 5 & & 3 & 5 & & & & & & & 4 & \\
\hline & $\mathrm{AF}$ & 6 & & 5 & 6 & & & & & & & 2 & 3 \\
\hline \multirow[t]{5}{*}{ Total } & $\mathrm{PN}$ & & & & & & & & & & & & \\
\hline & RA & & 1 & 2 & 4 & & 3 & 2 & 1 & 5 & 2 & 1 & 2 \\
\hline & $\mathrm{RH}$ & & 2 & & & & & & & 1 & 3 & & \\
\hline & $A C$ & 1 & & & 1 & 1 & 2 & & 1 & 4 & & & \\
\hline & $\mathrm{AF}$ & 2 & 3 & 2 & 2 & & 2 & 1 & 1 & 2 & 3 & & 2 \\
\hline
\end{tabular}

Obs: Os números dentro das células indicam a intensidade da correlação $(1=0,10$ a 0,$19 ; 2=0,20$ a 0,$29 ; 3=0,30$ a $0,39 \ldots$ e $8=0.80$ a 0.89 )

PN: relação partos/nascimentos

RA: Recursos ambulatoriais

$\mathrm{RH}$ : Recursos hospitalares

AC: Adesão ao PACS

AF: Adesão ao PSF

P102: Procedimentos 0707102

P103: Procedimentos 0707103

Para análise do desempenho no atendimento pré-natal foi construído o indicador de produtividade, calculado por porte de município e tendo como base o número de partos pagos pelo Sistema Único de Saúde (SUS), por local de residência dos municípios analisados. Os resultados apresentados na Tabela 3 mostram que a mediana de partos pagos pelo SUS por município de ocorrência, variou de 5,27 a 444,75 partos. Portanto esses seriam o numero de ciclos gravídicos puerperais naturais ocorridos nesses municípios para encontrar um registro completo de procedimentos preconizados pelo PHPN.
Tendo em vista a premissa de que o PSF seria uma condição facilitadora para a adesão dos municípios aos programas, observa-se na Tabela 4 que a adesão ao PSF não apresentou nenhuma associação quanto à adesão ou não ao PHPN, em qualquer dos portes de municípios.

A oferta existente nos municípios seria uma das condições facilitadoras para a implementação do PHPN. A Tabela 5 mostra que a região Sul apresenta a melhor oferta, mas que essa condição não influiu na adesão ao PHPN em nenhuma das regiões. 
A análise das distintas variáveis facilitadoras ao desempenho do PHPN é apresentada na Tabela 6, com as respectivas intensidades de correlação. Com base nessa análise de correlações, foram pré-selecionados 25 municípios no País.

A evasão ao acompanhamento pré-natal é apresentada na Figura 1, que mostra diferenciação nas taxas de evasão entre os distintos portes de município. As maiores taxas de evasão são observadas nas capitais.

Na Figura 2 apresenta-se a média dos principais indicadores de atenção: gestantes com seis consultas de pré-natal (MG6CON), gestantes com oito exames laboratoriais (MG8EX), gestantes com seis exames laboratoriais (MG6EX), gestantes imunizadas (MGIM) e gestantes com exame HIV (MGHIV), segundo região do País. Os maiores valores foram encontrados nas regiões Sul e Sudeste, provavelmente relacionado a maior oferta de serviços nessas regiões.

Os principais resultados da abordagem qualitativa baseada nas entrevistas semi-estruturadas são compatíveis com a maioria dos achados da abordagem quantitativa, a saber: as gestantes são captadas com até 120 dias de gestação, realizam as seis consultas durante o pré-natal, realizam a primeira bateria de exames laboratoriais e a maioria é imunizada contra o tétano. Nessa abordagem foi possível identificar as seguintes fragilidades do PHPN: a falta de integração funcional efetiva entre o pré-natal e o parto, a reduzida realização da segunda bateria de exames complementares no terceiro trimestre de gravidez, e a escassa realização da consulta de puerpério.

A diretriz da humanização do parto não vem sendo cumprida. As visitas aos hospitais preconizadas pelo Programa não ocorrem, provavelmente pela não concretização das referências específicas. Isso pode ser em razão da valorização do conhecimento prévio da referência hospitalar pelas gestantes, que representa importante fator de segurança e confiança.

A análise crítica do Sisprenatal indica que o sistema é amigável; acessível; possui coerência interna; tem potencial de instrumentalizar o gerenciamento do
PHPN a partir dos relatórios gerenciais que emite; quando utilizado com oportunidade, o Sistema não retrata de forma integral a realidade do atendimento realizado no local de atenção; e finalmente, o Sistema apresenta uma defasagem no tempo e no quantitativo dos dados disponíveis no sistema em nível local, quando comparado com o central.

As principais recomendações formuladas ao PHPN, com base nos resultados desta avaliação foram:

1. definição de um mecanismo funcional efetivo de integração entre o pré-natal e o parto, acompanhado de um instrumento administrativo de controle e de remuneração, seja por meio de uma autorização específica ou por um plano de meta para essa integração;

2. contextualização da consulta do puerpério, com flexibilização do intervalo de tempo definido entre o parto e o momento de contato da puérpera com o serviço. As atividades de controle do pós-parto devem ser decorrentes das oportunidades de contato já existentes com o serviço, tais como o controle do recém-nascido, a consulta de planejamento familiar ou a visita domiciliar;

3. focalização da segunda bateria de exames no diagnóstico precoce da sífilis, flexibilizando os outros exames laboratoriais para indicação médica. Dessa forma, a norma técnica poderia ser relativizada, permitindo a pactuação com o sistema local do retorno do resultado de forma mais rápida e com factibilidade de atuação (tratamento, se necessário), prévia ao nascimento;

4. modificação da atual lógica de financiamento para metas de integração do pré-natal e parto: realização do controle pós-parto na oportunidade de contato da puérpera com o serviço de saúde e priorização do teste para sífilis na solicitação normativa de exames no terceiro trimestre, possibilitando potencializar o impacto induzido pelo estímulo financeiro ao PHPN. 